\title{
Scattering of light by a large, densely packed agglomerate of small silica spheres
}

\section{Väisänen, T.}

2020-04-01

Väisänen , T , Markkanen , J , Hadamcik , E , Renard , J-B , Lasue , J , Levasseur-Regourd , A C , Blum , J \& Muinonen , K 2020 , ' Scattering of light by a large, densely packed agglomerate of small silica spheres ' , Optics Letters , vol. 45 , no. 7 , pp. 1679-1682 . https://doi.org/10.1364/OL.382

http://hdl.handle.net/10138/328623

https://doi.org/10.1364/OL.382240

acceptedVersion

Downloaded from Helda, University of Helsinki institutional repository.

This is an electronic reprint of the original article.

This reprint may differ from the original in pagination and typographic detail.

Please cite the original version. 


\title{
Scattering of light by a large, densely-packed agglomerate of small silica spheres
}

\author{
T. VÄISÄnen ${ }^{1,}$, J. MARKKANEN ${ }^{2}$, E. HAdAMCIK ${ }^{3}$, J.-B. RENARD ${ }^{4}$, J. LASUE ${ }^{5}$, A.C. \\ LeVASSEUR-REGOURD $^{6}$, J. BLUM ${ }^{7}$, AND K. MUINONEN ${ }^{1,8}$ \\ ${ }^{1}$ Department of Physics, University of Helsinki, P.O. Box 64, Gustaf Hällströmin katu 2, Helsinki FI-00014, Finland \\ ${ }^{2}$ Max Planck Institute for Solar System Research, Justus-von-Liebig-Weg 3, 37077 Göttingen, Germany \\ ${ }^{3}$ LATMOS/IPSL, UVSQ Université Paris-Saclay, Sorbonne Université, CNRS, Guyancourt, France \\ ${ }^{4}$ LPC2E-CNRS, 3A avenue de la Recherche Scientifique, 45071 Orléans cedex 2, France \\ ${ }^{5}$ IRAP, Université de Toulouse, CNRS, CNES, UPS, 9 avenue Colonel Roche, 31400 Toulouse, France \\ ${ }^{6}$ LATMOS, Sorbonne Univ., CNRS, UVSQ, Campus Pierre et Marie Curie, 4 place Jussieu, 75005 Paris, France \\ ${ }^{7}$ Institut für Geophysik und extraterrestrische Physik, Technische Universität Braunschweig, Mendelssohnstr. 3, 38106 Braunschweig, Germany \\ ${ }^{8}$ Finnish Geospatial Research Institute FGI, National Land Survey of Finland, Geodeetinrinne 2, FI-02430 Masala, Finland \\ *Corresponding author: timo.h.vaisanen@helsinki.fi
}

Compiled June 9, 2020

We model the measured phase function and degree of linear polarization of a macroscopic agglomerate made of micron-scale silica spheres using the methodology of multiple scattering. In the laboratory work, the agglomerate is produced ballistically, characterized by scanning electron microscopy, and measured with the PROGRA $^{2}$ instrument to obtain the light scattering properties. The model phase function and degree of polarization are in satisfactory agreement with the experimental data. To our best knowledge, this is the first time the degree of linear polarization has been modeled well for a large, densely-packed agglomerate composed of small particles with known sizes and shapes. The study emphasizes the relevance of the degree of linear polarization and gives insights into the effects of particle aggregation on the scattering characteristics. () 2020 Optical Society of America

http://dx.doi.org/10.1364/ao.XX.XXXXXX

Scattering and absorption of light by a particulate medium provides information about the physical properties of the medium and its particles. Computing scattering by a medium with millions of particles is a challenging problem due to the computational complexity. Numerical methods that solve Maxwell's equations exactly in the asymptotic sense, e.g., the fast superposition T-matrix method (FaSTMM, [1]), are limited to media typically smaller than some tens of wavelengths. Thus, approximations have been formulated to simulate scattering by dense particulate media such as snow (Dense Media Radiative Transfer, DMRT) [2] and planetary regoliths [3, 4]. One of the most recent approximations is Radiative Transfer with Reciprocal Transactions $\left(\mathrm{R}^{2} \mathrm{~T}^{2}\right)$. It has been shown to extend the applicability of radiative transfer to the dense medium [5-7] by comparing the $\mathrm{R}^{2} \mathrm{~T}^{2}$ computations to the computations with the
FaSTMM and Radiative Transfer with Coherent Backscattering methods (RT-CB). In comparison, the FaSTMM required around 600 days in serial CPU time to compute ensemble-averaged light scattering characteristics from a dense random medium made of around 31000 particles, whereas the entire $R^{2} T^{2}$ procedure took only 7 days [5].

The $\mathrm{R}^{2} \mathrm{~T}^{2}$ has been used to model the nucleus and dust particles in the coma of Comet 67P/Churyumov-Gerasimenko [8, 9], as well as a levitating analog sample [10]. Here, we use the $\mathrm{R}^{2} \mathrm{~T}^{2}$ to reproduce computationally the light scattering from a centimeter-sized silica sample, measured with the PROGRA ${ }^{2}$ instrument [11-13]. The sample had controlled physical parameters, such as the particle size distribution, shape, and total volume fraction of $v=0.15$ [14]. From scanning electron microscope (SEM) images (Fig. 1), the particles were verified to be mostly spherical, and their radii were found to follow a Gaussian size distribution $\mathcal{N}(0.725[\mu \mathrm{m}], 0.03[\mu \mathrm{m}])[11]$. In addition, the light scattering characteristics of the powder that was used to prepare the agglomerate were measured for levitating particles with the PROGRA $^{2}$ instrument. The refractive index of the material was then derived by fitting Lorenz-Mie light scattering characteristics to the measured characteristics [11]. In order to computationally model the light scattering characteristics of the sample, we start by examining the determination of the refractive index from the same measurements.

First, volume elements of radius $R=1.0 \mu \mathrm{m}$ are generated using spherical particles so that the centers of the spheres are within the volume elements. The volume elements are culled from large periodic boxes packed to the volume fraction of $55 \%$ with the spheres whose size distribution obeys $\mathcal{N}(0.725[\mu \mathrm{m}]$, $0.03[\mu \mathrm{m}])$. The small volume elements mimic small clusters of levitating particles because some of the particles are clustered or even sintered together (see Fig. 2 in [11]). The ensembleaveraged light scattering characteristics of the volume elements are then solved by using the FaSTMM by varying the complex refractive index. The results and the measurements are com- 


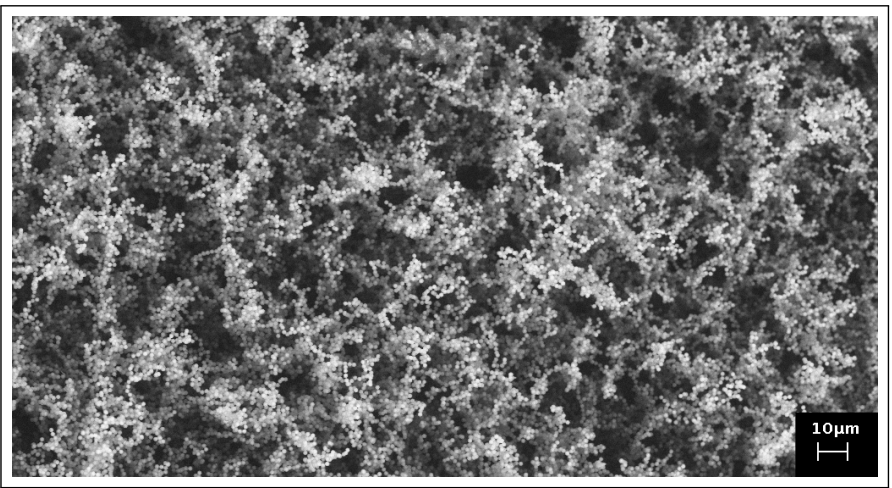

Fig. 1. Scanning electron microscope image of the $\mathrm{SiO}_{2}$ agglomerate sample. More images, including those of the entire cm-scale sample, are available in $[11,12,14]$.

pared in Fig. 2 that shows the phase function (scattering matrix element $S_{11} ;[15]$ ) normalized at $90^{\circ}$ scattering angle (spherical polar angle measured from the forward direction) and the degree of linear polarization (scattering matrix element ratio $-S_{12} / S_{11}$; [15]). The determination of the refractive index is difficult because most of the features are hard to match, especially with the phase functions. By comparing the degree of linear polarization near the forward and backward scattering directions, the simulated values are seen to be closer to the measured values near the forward direction. The most notable feature is the missing positive polarization near $150^{\circ}$. The refractive index $m=1.48+110^{-5}$ is chosen because it produces a match with the distinctive feature found near $20^{\circ}$ in the measurements of the polarization. In [11], the refractive index was determined to be $m=1.48+\mathrm{i} 10^{-4}$ for the wavelength of $\lambda=632.8 \mathrm{~nm}$ with Lorenz-Mie theory, and the results are similar to those in Fig. 2 (see Fig. 10 in [11]). In comparison, the manufacturer of the silica spheres reported the refractive index of $m=1.5$. In different measurements made for larger spheres of the same material, the refractive index of $1.30+\mathrm{i} 0.08$ was determined for the material, attributed to the porosities reported by the manufacturer [12]. Fig. 2 and the work in [11] are also consistent with the work in [16], in which agglomerates of different sizes, consisting of the silica spheres with $m=1.48$ and radius $r=750 \mathrm{~nm}$, were measured and simulated with $\lambda=680 \mathrm{~nm}$. In [16], they also reported the missing positive polarization near $150^{\circ}$ in their simulations, although the measurements showed a strong positive polarization signature there. They attributed the difference to particle inhomogeneities [17].

The agglomerate can now be modeled with the $\mathrm{R}^{2} \mathrm{~T}^{2}$. The $\mathrm{R}^{2} \mathrm{~T}^{2}$ is a radiative transfer method that is extended to work with dense random particulate media by incorporating so-called incoherent scattering in the frequency domain [18-22]. The incoherent electric field $\mathbf{E}^{\text {sca,ic }}$ can be extracted by subtracting the ensemble-averaged total scattered field or coherent field $\mathbf{E}^{\text {sca,c }}$ from the scattered field $\mathrm{E}^{\mathrm{sca}}$. In the $\mathrm{R}^{2} \mathrm{~T}^{2}$, the single particles are replaced with the incoherent volume elements [7]. The volume elements are generated in the same way as mentioned above, but, in order to utilize larger numbers of particles, the size of the volume element is chosen to be $R=3 \mu \mathrm{m}$. Two different volume fractions are treated here, i.e., $v=0.15$ and $v=0.55$. The scattered incoherent electric fields of the volume elements are then solved using semi-analytical methods such as the FaSTMM, which can be used to generate the input (extinction mean free path length,

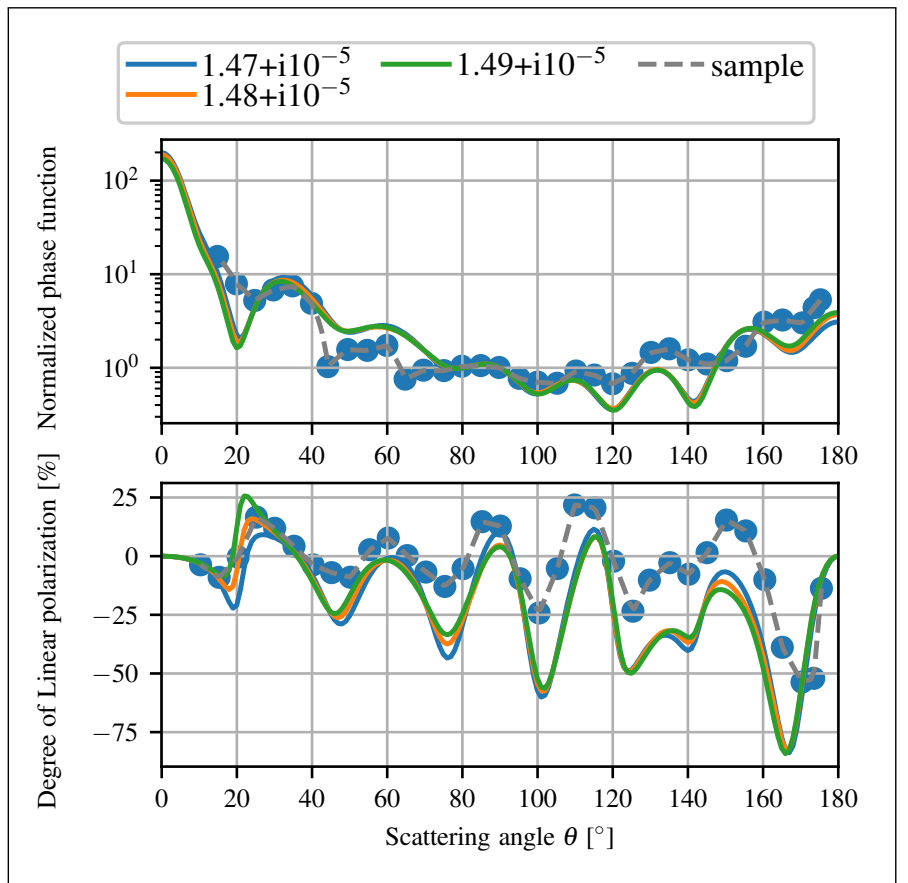

Fig. 2. Comparison of normalized phase function (at $90^{\circ}$ ) and the degree of linear polarization for levitating particles (measurements, bullets) and small clusters of spherical particles with varying refractive indices (computations, solid lines). Empirical phase function and degree of polarization are also depicted (dashed line).

albedo, effective refractive indices, and incoherent scattering matrix) required for the $\mathrm{R}^{2} \mathrm{~T}^{2}$.

Here, we follow the procedure from [8] in which the $\mathrm{R}^{2} \mathrm{~T}^{2}$ is approximated with the ray tracer SIRIS $[7,23,24]$ which has geometric optics capabilities. SIRIS can be used to model differently shaped diffusely scattering media, but does not have the coherent backscattering capability. In order to create a planar agglomerate for the simulation, the diffuse geometries in SIRIS are approximated by large sphere-like meshes (mean radius $R \approx 2 \mathrm{~mm}$ ) that are illuminated by a narrow beam $\left(R_{\text {beam }}=0.2 \mathrm{~mm}\right)$ constrained into the center of the medium. Thereby, the global scale structure of the mesh will not affect the simulation, but still, the simulation continues to take into account the local scale surface structures. The final output is smoothened by ensemble-averaging using the simulation data from multiple runs of SIRIS that had different geometries as input. Three completely different geometries are used: a sphere, a Gaussian random sphere (GRS) [25], and a sphere with the inner structure of the Gaussian random field (GRFS, Fig. 3). The GRFS is obtained by carving a spherical volume from a sample of Gaussian field [26] and converting the field data to a triangulated mesh with the marching cubes algorithm [27]. The inner parts of the GRFS ( $R_{\text {inner }}=1 \mathrm{~mm}$ ) is converted to a solid sphere in favor of reducing the triangle count and speeding up the computation. The Gaussian random field has been applied to the light scattering problems before, e.g., in [28, 29]. The longest task would take around $3.5+27$ days ( 4 million rays) to create input and finish the ray-tracing with a single core. The computations and measurements are shown in Fig. 4 that contains the phase function (normalized in terms of integrated area) and the degree of linear polarization as a function of the scattering angle. 


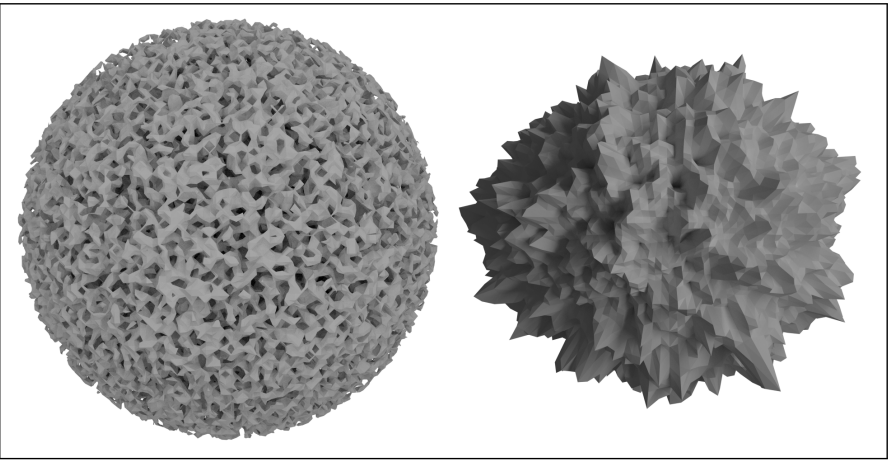

Fig. 3. Samples of Gaussian random field (left) and Gaussian random sphere geometries (right). Their surface elements are invoked as models for the measurement sample in Fig. 4.

Due to the fact that the measured data range from $100^{\circ}$ to $175^{\circ}$, thus not reaching the angles near backscattering, the coherent backscattering modeling is omitted.

By only looking at the overall phase function in Fig. 4, we could argue that the $v=0.15$ volume element produces a good match with the measurement. The phase function has a distinctive bump at $155^{\circ}$, which in the measurement is near $160^{\circ}$. The level of backscattering is slightly lower than that in the measurements, but this can be mitigated with the increase of the shadowing effect [4]. It is the degree of linear polarization that reveals a difference between the simulation and the measurement. The polarization shows the same $5^{\circ}$ shift as the phase function (Fig. 2 shows the same shift), and there is a uniform difference of around 2-4 percentage points. The match in the degree of linear polarization is improved by using the $v=0.55$ volume element and the difference is only 1-3 percentage points. However, the $5^{\circ}$ shift to the left is still present. For the $v=0.55$ volume element, the phase function is also flatter than that for the $v=0.15$ volume element. This is seen in the loss of the distinctive bump near $\theta \approx 160^{\circ}$ that is more prominent with the GRFS samples. In general, similar results are obtained with the GRS and GRFS models which shows that small changes in the geometry do not impact the results substantially. Thus, the focus can be on the properties of the particles. For changes due to the overall shape of the finite medium, compare "Sphere, $v=0.55$ " with "GRS, $v=0.55$ " in Fig. 4.

The use of the $v=0.55$ volume element improves the modeling. The dense volume element does not contradict the measured volume fraction of $v=0.15$ because the SEM image (see Fig. 1 or Fig. 4 in [11]) shows that the spheres have formed threads of clumps that are closely connected probably by sintering, forming high-density localities separated by empty spaces. Moreover, the particles in the $v=0.15$ volume elements were uniformly placed, whereas Fig. 1 shows aggregated structures. Modeling the aggregated structures can introduce the bump near $\theta \approx 160^{\circ}$ due to the introduction of the sparse regions to the dense volume element, but can lower the negative polarization at $\theta \approx 165^{\circ}$. The dense volume element is still insufficient as is seen in Fig. 2 where prominent positive polarization is again missing.

It is interesting to notice that most of the features seen in the scattering characteristics of the agglomerate are also visible for the levitating sample (cf. measurements in Figs. 2 and 4). The same can be concluded in the case of the computations: the features are aligned. This means that the features are consis-

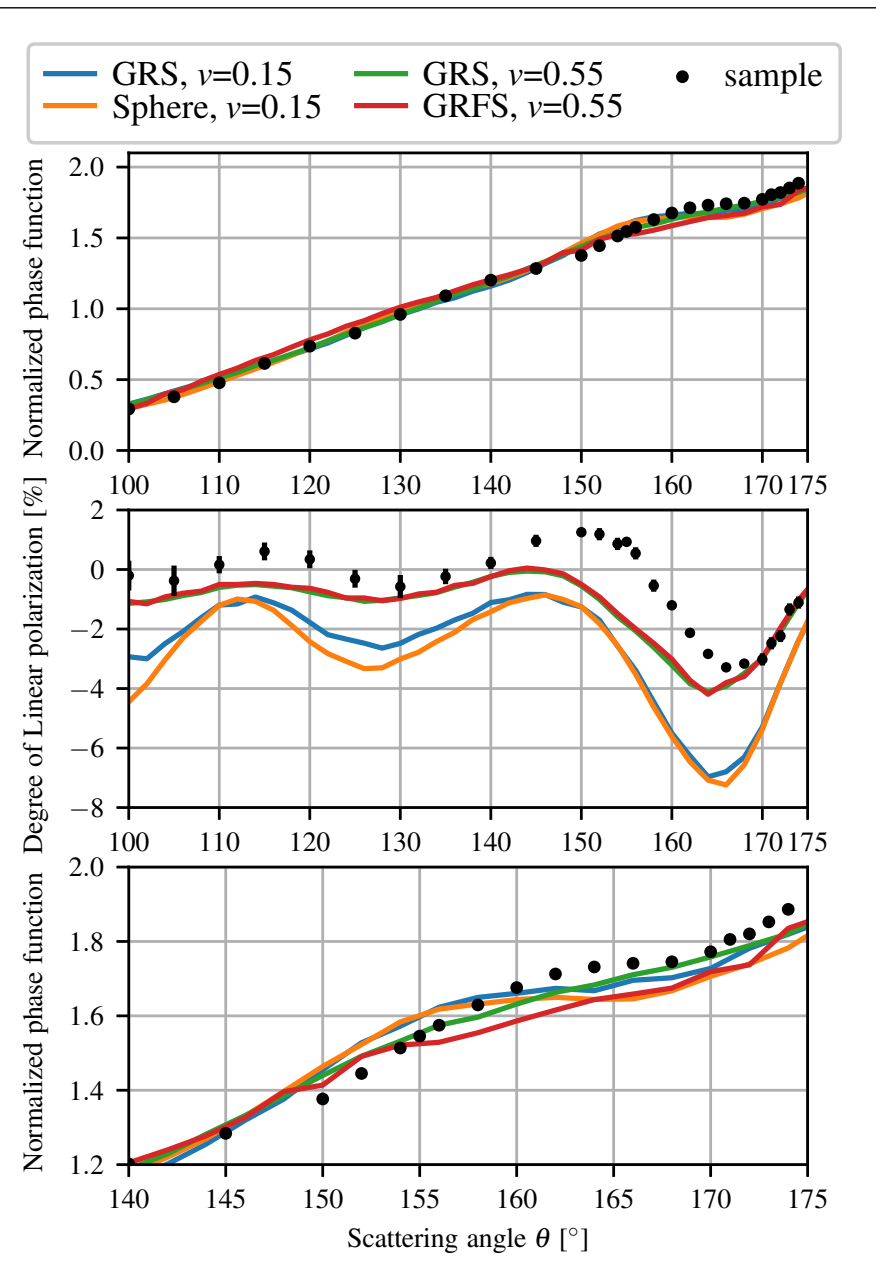

Fig. 4. The measured and modeled light scattering characteristics of the agglomerate. The refractive index is $m=1.48+i 10^{-} 5$, while the volume fraction and the shape of the media are varied. The measurements include the error bars.

tent between the measurements and the computation, and by achieving a better match with the scattering characteristics of the levitating sample, the results for the agglomerate should improve. In order to test this, we generated an empirical volume element using the measured scattering matrix elements of the levitating sample $M_{11}^{l}$ and $-M_{12}^{l} / M_{11}^{l}$ and using the computed matrix elements for the $v=0.55$ volume element $S_{11}^{v}$ and $-S_{12}^{v} / S_{11}^{v}$. First, the empirical volume element phase function $S_{11}^{v e}$ is obtained by scaling $M_{11}^{l}$ with the coefficient $S_{11}^{v} / S_{11}^{s}$, where $S_{11}^{S}$ refers to the average phase function of simulated levitating particles: $S_{11}^{v e} \propto M_{11}^{l} S_{11}^{v} / S_{11}^{s}$. Then, a scaling factor $\beta \approx 0.32$ for $-M_{12}^{l} / M_{11}^{l}$ is found by minimizing the root-meansquare difference between $-\beta S_{12}^{s} / S_{11}^{s}$ and $-S_{12}^{v} / S_{11}^{v}$. The resulting degree of linear polarization for the empirical volume element is then $-S_{12}^{v e} / S_{11}^{v e}=-\beta M_{12}^{l} / M_{11}^{l}$. The other matrix elements are assumed to be zero except for $S_{21}^{v e}=S_{12}^{v e}, S_{22}^{v e}=S_{11}^{v e}$, and $S_{33}^{v e}=S_{44}^{v e}= \pm S_{11}^{v e} \sqrt{1-\left[S_{12}^{v e} / S_{11}^{v e}\right]^{2}}$ (positive sign for scattering angles $<90^{\circ}$ ). The empirical scattering matrix is then used as input for $\mathrm{R}^{2} \mathrm{~T}^{2}$, by keeping the rest of the volume element parameters unchanged. The results in Fig. 5 show that the empirical volume element removes the differences due to the 


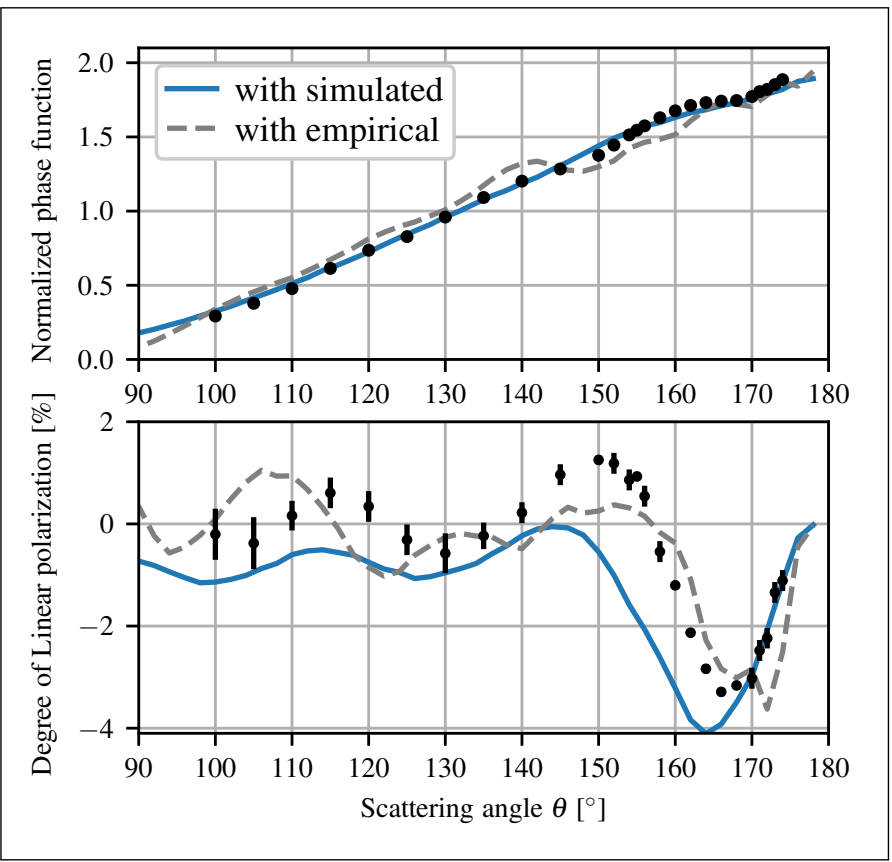

Fig. 5. The measured and modelled light scattering characteristics of the agglomerate with different volume elements. The result with the simulated volume element is the " $v=0.55$, GRS" case from Fig. 4.

original simulation, such as the $5^{\circ}$ shift and the missing positive polarization. The results indicate that, once correct modeling is obtained for the levitating sample (Fig. 2), the agglomerate scattering characteristics will also be modeled correctly.

Even though there are differences between the measurements and the computational results, the examination of the input and the results show that the modeling and the measurements are consistent. By improving the characterization of the levitating particles, a better match would be obtained with the $\mathrm{R}^{2} \mathrm{~T}^{2}$ for the agglomerate. In a future study, improved modeling for the levitating particles could involve sintered particles, small aggregates, or particles with other kinds of deformations, and a distribution of the refractive indices. Simulating the entire volume element for the $\mathrm{R}^{2} \mathrm{~T}^{2}$ with irregular particles is computationally challenging but not impossible, especially when modeling the scattering by the levitating particles at the same time. The present results are promising. With the empirical volume element (Fig. 5), the normalized root-mean-square error in modeling the agglomerate phase function is less than $7 \%$, and the degree of linear polarization is within 1.5 percentage point of the measurements. With the simulated volume element, the respective numbers are $2.5 \%$ and 3 percentage points. The study stresses the importance of sample characterization in light scattering measurements. Finally, the study emphasizes the importance of polarization: the physical characteristics are hard to retrieve from the phase function only.

\section{ACKNOWLEDGMENTS AND FUNDING}

Research by T.V. and K.M. partially supported by the Academy of Finland grant 1325805. J.M. was supported by ERC Grant No. 757390. J.B. thanks the Deutsches Zentrum für Luft- und Raumfahrt (DLR) for support under grants 50WM1236, 50WM1536 and 50WM1846. The PROGRA ${ }^{2}$ instrument was partly funded by the French Space Agency CNES. Computational resources were provided by CSC-IT Centre for Science Ltd., Finland, and Finnish Grid and Cloud Infrastructure, (urn:nbn:fi:researchinfras-2016072533).

\section{DISCLOSURES}

The authors declare no conflicts of interest.

\section{REFERENCES}

1. J. Markkanen and A. J. Yuffa, J. Quant. Spectrosc. Radiat. Transf. 189, 181 (2017).

2. L. Tsang, C.-T. Chen, A. T. C. Chang, J. Guo, and K.-H. Ding, Radio Sci. 35, 731 (2000).

3. B. Hapke, J. Geophys. Res. Solid Earth 86, 3039 (1981).

4. K. Lumme, J. I. Peltoniemi, and W. M. Irvine, Transp. Theory Stat. Phys. 19, 317 (1990).

5. K. Muinonen, J. Markkanen, T. Väisänen, J. Peltoniemi, and A. Penttilä, Opt. Lett. 43, 683 (2018).

6. J. Markkanen, T. Väisänen, A. Penttilä, and K. Muinonen, Opt. Lett. 43, 2925 (2018).

7. T. Väisänen, J. Markkanen, A. Penttilä, and K. Muinonen, PLOS ONE 14, 1 (2019).

8. J. Markkanen, J. Agarwal, T. Väisänen, A. Penttilä, and K. Muinonen, Astrophys. J. Lett. 868 (2018).

9. J. Markkanen and J. Agarwal, Astron. \& Astrophys. (2019).

10. K. Muinonen, T. Väisänen, J. Martikainen, J. Markkanen, A. Penttilä, M. Gritsevich, J. Peltoniemi, J. Blum, J. Herranen, G. Videen, G. Maconi, P. Helander, A. Salmi, I. Kassamakov, and E. Haeggström, J. Vis. Exp. (2019).

11. E. Hadamcik, J.-B. Renard, J. Lasue, A.C. Levasseur-Regourd, J. Blum, and R. Schraepler, J. Quant. Spectrosc. Radiat. Transf. 106, 74 (2007).

12. E. Hadamcik, J.-B. Renard, A.C. Levasseur-Regourd, J. Lasue, G. Alcouffe, and M. Francis, J. Quant. Spectrosc. Radiat. Transf. 110, 1755 (2009).

13. A.C. Levasseur-Regourd, J.-B. Renard, Y. Shkuratov, and E. Hadamcik, "Laboratory studies," in Polarimetry of Stars and Planetary Systems, L. Kolokolova, J. Hough, and A.C. Levasseur-Regourd, eds. (Cambridge University Press, 2015), pp. 62-80.

14. J. Blum and R. Schräpler, Phys. Rev. Lett. 93, 115503 (2004).

15. C. F. Bohren and D. R. Huffman, Absorption and scattering of light by small particles (New York: Wiley, 1983).

16. G. Wurm, H. Relke, J. Dorschner, and O. Krauß, J. Quant. Spectrosc. Radiat. Transf. 89, 371 (2004).

17. G. Wurm, H. Relke, and J. Dorschner, The Astrophys. J. 595, 891 (2003).

18. A. Ishimaru, Wave Propagation and Scattering in Random Media, An IEEE OUP classic reissue (Wiley, 1999).

19. L. Tsang, J. Kong, and R. Shin, Theory of Microwave Remote Sensing, Wiley Series in Remote Sensing and Image Processing (Wiley, 1985).

20. L. Tsang, C. E. Mandt, and K. H. Ding, Opt. Lett. 17, 314 (1992).

21. L. M. Zurk, L. Tsang, and D. P. Winebrenner, Radio Sci. 31, 803 (1996).

22. M. I. Mishchenko, L. D. Travis, and A. A. Lacis, Multiple Scattering of Light by Particles: Radiative Transfer and Coherent Backscattering (Cambridge University Press, Cambridge, 2006).

23. K. Muinonen, T. Nousiainen, H. Lindqvist, O. Muñoz, and G. Videen, J. Quant. Spectrosc. Radiat. Transf. 110, 1628 (2009).

24. H. Lindqvist, J. Martikainen, J. Räbinä, A. Penttilä, and K. Muinonen, J. Quant. Spectrosc. Radiat. Transf. 217, 329 (2018).

25. K. Muinonen, T. Nousiainen, P. Fast, K. Lumme, and J. Peltoniemi, J. Quant. Spectrosc. Radiat. Transf. 55, 577 (1996).

26. S. Müller and L. Schüler, "Geostat-framework/gstools: Bouncy blue," (2019).

27. W. E. Lorensen and H. E. Cline, SIGGRAPH Comput. Graph. 21, 163-169 (1987)

28. J. I. Peltoniemi, J. Quant. Spectrosc. Radiat. Transf. 50, 655 (1993).

29. Y. Grynko and Y. Shkuratov, J. Quant. Spectrosc. Radiat. Transf. 78, 319 (2003). 


\section{FULL REFERENCES}

1. J. Markkanen and A. J. Yuffa, "Fast superposition t-matrix solution for clusters with arbitrarily-shaped constituent particles," J. Quant. Spectrosc. Radiat. Transf. 189, $181-188$ (2017).

2. L. Tsang, C.-T. Chen, A. T. C. Chang, J. Guo, and K.-H. Ding, "Dense media radiative transfer theory based on quasicrystalline approximation with applications to passive microwave remote sensing of snow," Radio Sci. 35, 731-749 (2000).

3. B. Hapke, "Bidirectional reflectance spectroscopy: 1. theory," J. Geophys. Res. Solid Earth 86, 3039-3054 (1981).

4. K. Lumme, J. I. Peltoniemi, and W. M. Irvine, "Diffuse reflection from a stochastically bounded, semi-infinite medium," Transp. Theory Stat. Phys. 19, 317-332 (1990).

5. K. Muinonen, J. Markkanen, T. Väisänen, J. Peltoniemi, and A. Penttilä, "Multiple scattering of light in discrete random media using incoherent interactions," Opt. Lett. 43, 683-686 (2018).

6. J. Markkanen, T. Väisänen, A. Penttilä, and K. Muinonen, "Scattering and absorption in dense discrete random media of irregular particles," Opt. Lett. 43, 2925-2928 (2018).

7. T. Väisänen, J. Markkanen, A. Penttilä, and K. Muinonen, "Radiative transfer with reciprocal transactions: Numerical method and its implementation," PLOS ONE 14, 1-24 (2019).

8. J. Markkanen, J. Agarwal, T. Väisänen, A. Penttilä, and K. Muinonen, "Interpretation of the phase functions measured by the osiris instrument for comet 67p/churyumov-gerasimenko," Astrophys. J. Lett. 868 (2018).

9. J. Markkanen and J. Agarwal, "Scattering, absorption, and thermal emission by large cometary dust particles: Synoptic numerical solution," Astron. \& Astrophys. (2019).

10. K. Muinonen, T. Väisänen, J. Martikainen, J. Markkanen, A. Penttilä, M. Gritsevich, J. Peltoniemi, J. Blum, J. Herranen, G. Videen, G. Maconi, P. Helander, A. Salmi, I. Kassamakov, and E. Haeggström, "Scattering and absorption of light in planetary regoliths," J. Vis. Exp. (2019).

11. E. Hadamcik, J.-B. Renard, J. Lasue, A.C. Levasseur-Regourd, J. Blum, and R. Schraepler, "Light scattering by low-density agglomerates of micron-sized grains with the progra2 experiment," J. Quant. Spectrosc. Radiat. Transf. 106, $74-89$ (2007).

12. E. Hadamcik, J.-B. Renard, A.C. Levasseur-Regourd, J. Lasue, G. Alcouffe, and M. Francis, "Light scattering by agglomerates: Interconnecting size and absorption effects (progra2 experiment)," J. Quant. Spectrosc. Radiat. Transf. 110, 1755-1770 (2009).

13. A.C. Levasseur-Regourd, J.-B. Renard, Y. Shkuratov, and E. Hadamcik, "Laboratory studies," in Polarimetry of Stars and Planetary Systems, L. Kolokolova, J. Hough, and A.C. Levasseur-Regourd, eds. (Cambridge University Press, 2015), pp. 62-80.

14. J. Blum and R. Schräpler, "Structure and mechanical properties of high-porosity macroscopic agglomerates formed by random ballistic deposition," Phys. Rev. Lett. 93, 115503 (2004).

15. C. F. Bohren and D. R. Huffman, Absorption and scattering of light by small particles (New York: Wiley, 1983).

16. G. Wurm, H. Relke, J. Dorschner, and O. Krauß, "Light scattering experiments with micron-sized dust aggregates: results on ensembles of sio2 monospheres and of irregularly shaped graphite particles," J. Quant. Spectrosc. Radiat. Transf. 89, 371 - 384 (2004).

17. G. Wurm, H. Relke, and J. Dorschner, "Experimental study of light scattering by large dust aggregates consisting of micron-sized SiO2monospheres," The Astrophys. J. 595, 891-899 (2003).

18. A. Ishimaru, Wave Propagation and Scattering in Random Media, An IEEE OUP classic reissue (Wiley, 1999).

19. L. Tsang, J. Kong, and R. Shin, Theory of Microwave Remote Sensing, Wiley Series in Remote Sensing and Image Processing (Wiley, 1985).

20. L. Tsang, C. E. Mandt, and K. H. Ding, "Monte carlo simulations of the extinction rate of dense media with randomly distributed dielectric spheres based on solution of maxwell's equations," Opt. Lett. 17, 314316 (1992).

21. L. M. Zurk, L. Tsang, and D. P. Winebrenner, "Scattering properties of dense media from monte carlo simulations with application to active remote sensing of snow," Radio Sci. 31, 803-819 (1996).
22. M. I. Mishchenko, L. D. Travis, and A. A. Lacis, Multiple Scattering of Light by Particles: Radiative Transfer and Coherent Backscattering (Cambridge University Press, Cambridge, 2006).

23. K. Muinonen, T. Nousiainen, H. Lindqvist, O. Muñoz, and G. Videen, "Light scattering by Gaussian particles with internal inclusions and roughened surfaces using ray optics," J. Quant. Spectrosc. Radiat. Transf. 110, 1628 - 1639 (2009).

24. H. Lindqvist, J. Martikainen, J. Räbinä, A. Penttilä, and K. Muinonen, "Ray optics for absorbing particles with application to ice crystals at near-infrared wavelengths," J. Quant. Spectrosc. Radiat. Transf. 217, $329-337$ (2018).

25. K. Muinonen, T. Nousiainen, P. Fast, K. Lumme, and J. Peltoniemi, "Light scattering by gaussian random particles: Ray optics approximation," J. Quant. Spectrosc. Radiat. Transf. 55, 577 - 601 (1996).

26. S. Müller and L. Schüler, "Geostat-framework/gstools: Bouncy blue," (2019).

27. W. E. Lorensen and H. E. Cline, "Marching cubes: A high resolution 3d surface construction algorithm," SIGGRAPH Comput. Graph. 21, 163-169 (1987).

28. J. I. Peltoniemi, "Radiative transfer in stochastically inhomogeneous media," J. Quant. Spectrosc. Radiat. Transf. 50, 655 - 671 (1993).

29. Y. Grynko and Y. Shkuratov, "Scattering matrix calculated in geometric optics approximation for semitransparent particles faceted with various shapes," J. Quant. Spectrosc. Radiat. Transf. 78, 319 - 340 (2003). 